\title{
SOMATIC IMPLICATIONS IN HOSPITALIZED PATIENTS WITH CHRONIC SCHIZOPHRENIA
}

\author{
ANDREEA SĂLCUDEAN ${ }^{1}$, BIANCA-EUGENIA ŐSZ ${ }^{2}$, ELENA-GABRIELA STRETE ${ }^{3}$, \\ MONICA KISS ${ }^{4}$, MARIA-DORINA PASCA ${ }^{5}$, CRISTINA-RALUCA BODO ${ }^{6}$, MARIA-MELANIA \\ LICA $^{7}$, ANDREEA-GEORGIANA NAN ${ }^{8}$, MARIUS-CĂTĂLIN COSMA ${ }^{9}$, MARIANA TILINCA ${ }^{10}$

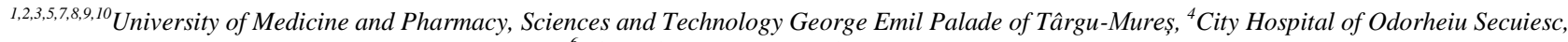 \\ ${ }^{6}$ Clinical County Hospital of Târgu-Mureș
}

Keywords: schizophrenia, somatic comorbidity, cardiovascular comorbidity, GAFS

\begin{abstract}
Schizophrenic patients have a life expectancy shorter than that of the general population. The multisystemic implication of the psychotic disease is evidenced by the high prevalence of somatic comorbidities associated with the illness. Patients with schizophrenia have a higher risk to develop somatic comorbidities due to their lifestyle changes, medication intake and poor medical assistance. We conducted a retrospective descriptive study in order to determine the prevalence of certain somatic conditions associated with schizophrenia as well as the characteristics of this particular group of patients.
\end{abstract}

\section{INTRODUCTION}

It is widely known that schizophrenia is a severe psychiatric disorder with multisystemic implication. Apart from the psychiatric burden, the excess mortality of the illness is a consequence of the association of different somatic comorbidities, especially cardiovascular and metabolic.

The life expectancy of this group of patients is shorter than that of the general population with approximately 15 to 30 years.(1)

More than $50 \%$ of the causes of death in patients with schizophrenia are due to somatic comorbidities, which are either diagnosed only after death or are poorly treated throughout life.(2) Another important cause of death (7-10\%) is represented by suicide, observed especially in patients with poor social support.(3)

Studies have shown that the pathogenesis of comorbidities involves a disorganized lifestyle, drug treatment by side effects and poor health care. Factors related to the characteristics of schizophrenia like negative symptoms such as anhedonia, emotional flattening or avolition contribute to the development of a sedentary lifestyle, with lack of physical activity and personal negligence. On the other hand, psychosocial factors and stigma can be responsible for the development of harmful behaviours, such as smoking, alcohol abuse or hypercaloric diet. Studies have noticed that social integration can be a marker for the treatment efficacy. The association of addictive disorders to schizophrenia aggravates the evolution of the psychotic illness. $(4,5)$

Regarding the drug treatment, atypical antipsychotics (such as olanzapine, quetiapine or risperidone) used to reduce symptoms include side-effects such as increased appetite and delayed feeling of satiety.(6)

The development of science has offered a new theory regarding the underlying pathogenesis of schizophrenia considering that the genetic link has an important impact on both psychiatric and somatic implications of schizophrenia.(7)

\section{AIM}

The aim of the study is to determine the prevalence of different somatic comorbidities affecting schizophrenic patients as well as particular characteristics such as: medication, number of admissions, co-occurring personality disorders, Global Assessment of Functioning Scale (GAF) scores.

\section{MATERIALS AND METHODS}

We performed a descriptive retrospective study (cross-sectional) including 97 patients admitted in the Psychiatry Department of Clinical County Hospital of TârguMureș during

01.01.2019-30.09.2020 and diagnosed with schizophrenia according to DSM V criteria.

In the study, there were included schizophrenic patients, both females or males, with no age limit, who associated at least one somatic comorbid disorder (cardiovascular, endocrine, nutrition and metabolism, gastrointestinal, neurological, dermatologic, respiratory, renal and genitourinary, cancer) at the time of the admission. Ninetyseven patients were eligible for the study.

In order to carry out the study, we analysed the information regarding the patients' pathologies found in the observation sheets, the psychiatric sheets, the psychological examination, the laboratory and imaging data, inter-clinical consultations and discharge papers.

The collected data was summarized in Microsoft Excel tables and analysed using the functions provided by the Microsoft Excel program: COUNT (IF / IFS), AVERAGE, IF (S).

We applied descriptive statistical analysis using the IBM SPSS Statistical program to calculate frequencies, averages, and distributions.

We used the GraphPad Prism 9 program to apply the necessary statistical tests (normality test; correlation coefficient of Spearman).

${ }^{8}$ Corresponding author: Andreea-Georgiana Nan, Bdul. Cetății, Nr. 1, Târgu-Mureș, Mureș, E-mail: nandree96@ yahoo.com, Phone: +40754576181 Article received on 16.07.2021 and accepted for publication on 02.09.2021 


\section{CLINICAL ASPECTS}

\section{RESULTS}

Patients' characteristics

Regarding the gender distribution, $74.23 \%$ of the studied patients were females, and only $25.77 \%$ were males. Average age of the group was 49.36 years. Age distribution indicated the highest prevalence of patients between 50-64 year ( $\mathrm{N}=43$ patients) followed by $26-49$ years group ( $\mathrm{N}=40$ patients).

Regarding the GAF scores (Global Assessment of Functioning Scale) $80 \%$ of patients had scores in the range of 41-50, indicating a serious impairment in social, professional or educational functioning (figure no. 1).

We found a high prevalence $(57.73 \%)$ of personality disorders among the patients, of which the most frequently encountered was the Schizoid Personality Disorder (SPD) (figure no. 2).

Figure no. 1. Distribution of patients according to GAF Scores

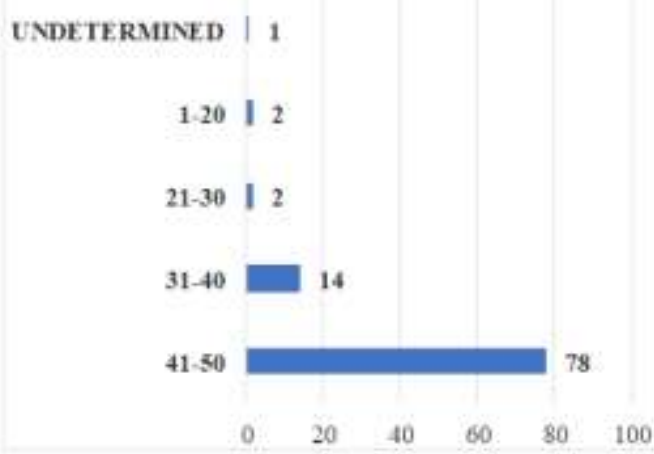

Figure no. 2. Distribution of personality disorders

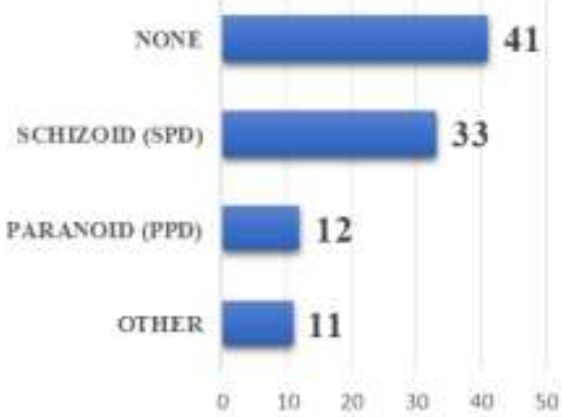

The average number of hospital admissions was 7.72. Most of the patients ( $\mathrm{n}=81)$ were hospitalized in the Psychiatry Department between 1-10 times during their life (figure no. 3).

Figure no. 3. Distribution by the number of admissions in the Psychiatry Department

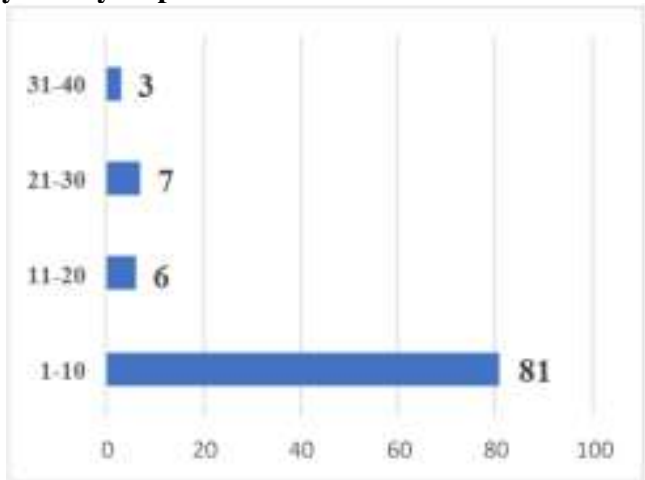

To determine if there is a correlation between the GAF score and the number of admissions, we applied the correlation test for nonparametric data. H0-null hypothesis- the value of the GAFS score does not lead to the change of the number of hospitalizations and the alternative hypothesis H1- the GAFS score influences the number of hospital admissions in the Psychiatry department. Spearman correlation coefficient: $r=$ 0.2083 - the two variables tend to increase or decrease together: a higher GAFS score leads to a bigger number of hospital admissions. $(\mathrm{p}=0.04$ - statistically significant) (table no. 1$)$.

Table no. 1. Correlation between GAF score and number of hospital admissions

\begin{tabular}{|l|l|}
\hline $\mathrm{r}$ & 0.2083 \\
\hline 95\% confidence interval & 0.002102 to 0.3974 \\
\hline $\mathrm{P}$ value & 0.0417 \\
\hline Exact or approximate P value? & Approximate \\
\hline Significant? (alpha $=0.05)$ & Yes \\
\hline
\end{tabular}

Somatic implication

Most of the patients included in the study $(n=41)$ had one somatic comorbidity (figure no. 4).

Figure no. 4. Distribution according to the number of somatic comorbidities

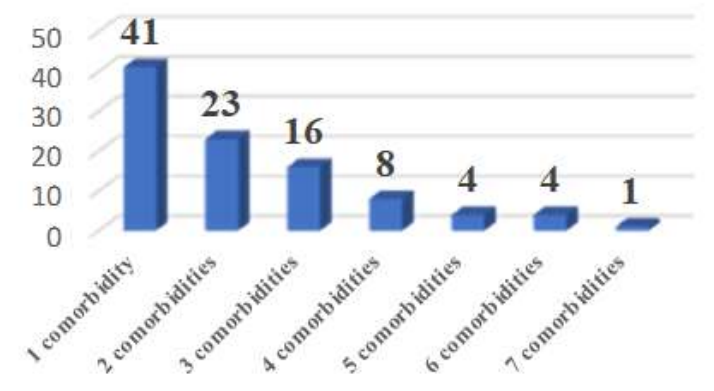

In order to determine if age influences the number of comorbidities, we used the Graph Pad analysis program and applied a nonparametric test to calculate the Spearman correlation coefficient. We considered the $\mathrm{H} 0$ - null hypothesis = no link between age and number of comorbidities, and $\mathrm{H1}$ alternative hypothesis $=$ the age influences the number of comorbidities. The test result was $\mathrm{r}=0.26$ - acceptable correlation. We will thus consider valid the alternative hypothesis (H1) -there is an acceptable link between age and number of comorbidities, (older patients tend to have more comorbidities) $p<0.05$-statistically significant (table no. 2).

Table no. 2. Correlation between age and number of comorbidities

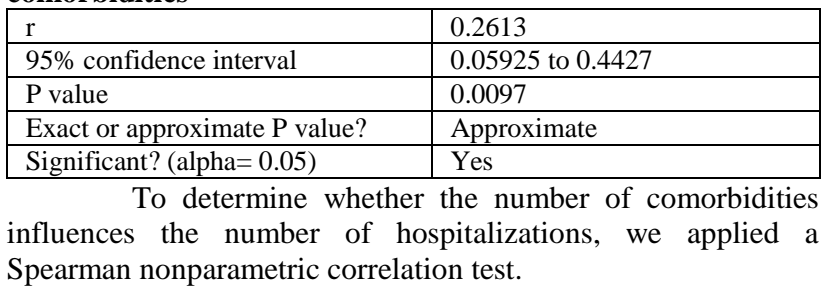

We considered the hypothesis H0-there is no correlation between the number of comorbidities and the number of hospitalizations and the alternative hypothesis $\mathrm{H1}$ the number of comorbidities influences the number of hospitalizations. The test result was $r=0.01664$ - weak / null correlation. Given that $\mathrm{p}=0.87>0.05$ - the test is statistically insignificant.

Therefore, we will consider the H0- hypothesis to be valid. There is no correlation between the number of somatic 


\section{CLINICAL ASPECTS}

comorbidities and the number of hospitalizations in the psychiatric service (table no. 3 ).

Table no. 3. Correlation between the number of comorbidities and the number of hospital admissions

\begin{tabular}{|l|l|}
\hline $\mathrm{r}($ Spearman) & 0.01664 \\
\hline 95\% confidence interval & $-0,1903$ to 0.2221 \\
\hline P value & 0,8722 \\
\hline Exact o approximate p value? & Approximate \\
\hline Significant? (alpha= 0.05$)$ & No \\
\hline
\end{tabular}

Increased cardiovascular morbidity $(\mathrm{N}=55$ out of 97 patients) was observed among the studied patients. The average age of the patients with cardiovascular pathologies was 49.17 years.

Endocrine, nutrition and metabolic diseases were the second most encountered comorbid conditions and affected 53 of the patients. Figure no. 5 describes the prevalence of different somatic comorbidities affecting the schizophrenic patients included in our study.

Figure no. 5. Number of patients with different somatic comorbidities

\section{SOMATICCOMORBIDITY}

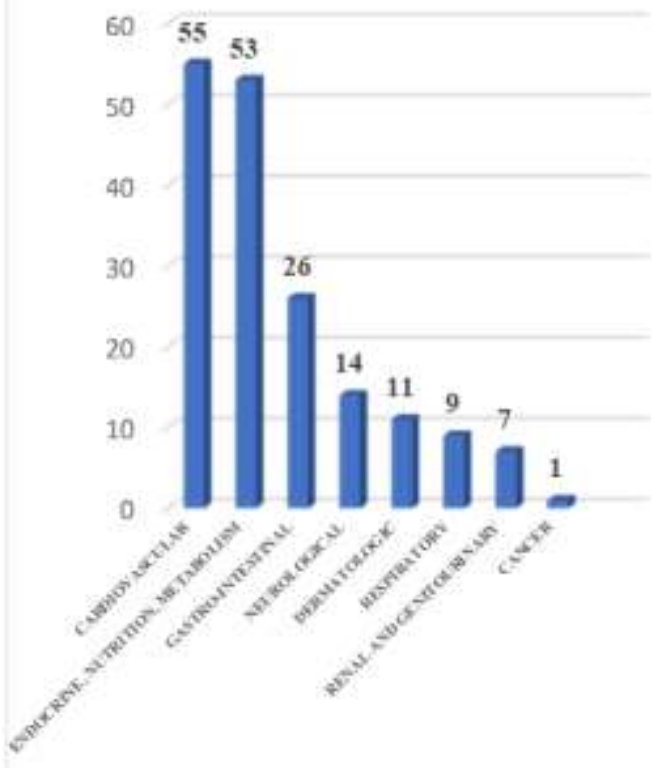

Figure no. 6. Atypical antipsychotic medication

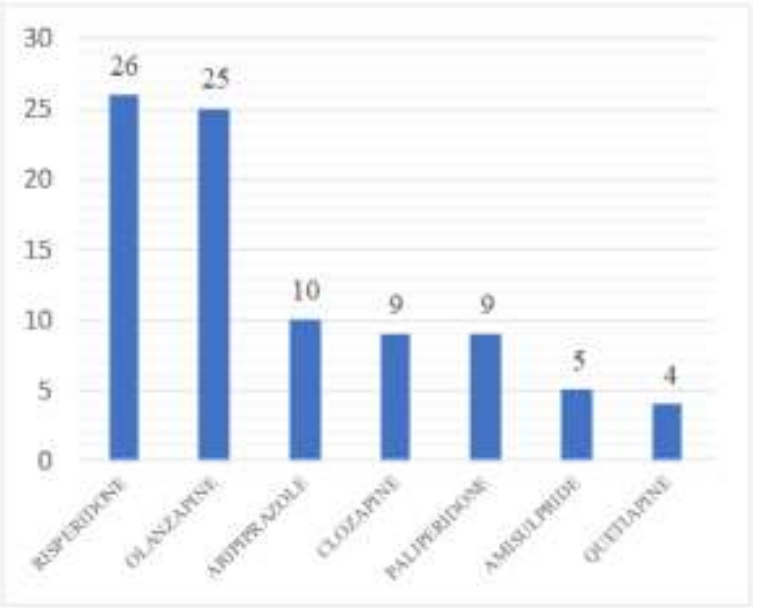

Treatment

Ninety one percent of the patients received atypical antipsychotics (figure no. 6), 1\% typical antipsychotics and $8 \%$ where currently under no antipsychotic medication.

Risperidone and olanzapine were the most used atypical antipsychotics among our studied group.

In addition to antipsychotic therapy, some patients have received other classes of drugs presented in table no. 4 and table no. 5 .

Table no. 4. Additional therapy

\begin{tabular}{|l|l|}
\hline \multicolumn{1}{|c|}{ Drug type } & Number of patients \\
\hline Benzodiazepines & 72 \\
\hline Mood stabilisers & 23 \\
\hline Antidepressants & 12 \\
\hline
\end{tabular}

Table no. 5. Benzodiazepine treatment

\begin{tabular}{|l|l|}
\hline \multicolumn{1}{|c|}{ Benzodiazepine } & Number of patients \\
\hline Alprazolam & 47 \\
\hline Clonazepam & 19 \\
\hline Nitrazepam & 9 \\
\hline Lorazepam & 2 \\
\hline Diazepam & 1 \\
\hline Bromazepam & 1 \\
\hline
\end{tabular}

\section{DISCUSSIONS}

Somatic burden of patients with schizophrenia represents a major challenge for the mental healthcare system. It is necessary to determine the characteristics of this particular group of patients in order to provide specific medical assistance and to reduce the mortality excess of the co-occurring somatic conditions.

More females than males were included in our study, even so we cannot conclude that the prevalence of this disease is different between genders due to the small number of patients involved in the study. Moreover, data regarding the prevalence reported in the literature is very controversial because the results are affected by the study design. Recently, more and more studies suggest that the prevalence is not different, only the age of diagnosis, women receiving a diagnosis a little later compared to men. For a review see (8).

Schizophrenic patients with associated somatic disorders had moderate and severe impairment in social, educational or occupational functioning.

We observed a positive correlation between an increased GAF score and a higher number of hospitalizations. This result could be due to the fact that schizophrenic patients with lower GAF scores are unaware of their psychiatric condition so they don't seek medical care. Illness denial is a frequent situation in psychiatric patients.(9)

The presence of a personality disorder was observed in $57.73 \%$ of patients, and the most common was schizoid personality disorder, followed by paranoid personality disorder. A research in Denmark highlights the negative impact on patients' long-term outcome of the association of schizophrenia with schizoid or other cluster A personality disorders.(10)

Our study indicated that the number of comorbidities increases with the age, so that older patients tend to have more comorbidities, just like in the general population.

Cardiovascular morbidity represented the major concern in terms of somatic illnesses. Studies in the literature mention the ascending nature of the prevalence of heart diseases among patients with psychiatric pathology, referring especially to the risks of atypical antipsychotic treatment with orexigenic side effects, disorganized lifestyle and a possible common genetic mechanism involved in the determinism of both conditions. $(7,11)$ 


\section{CLINICAL ASPECTS}

It is essential to focus on investigating the onset of the cardiovascular events to reduce the underdiagnosed or late diagnosed cases. A major concern is that the average age of patients with cardiovascular comorbidities was 49.17 years.

Endocrine, nutrition and metabolic abnormalities had a high prevalence among our studied group. Taking into account the high rates of atypical antipsychotics-based treatment, the metabolic abnormalities should be periodically investigated and prevented so that the overall risk could be minimised.

Regarding antipsychotic therapy, most patients (91\%) have atypical antipsychotic treatment with the predominance of risperidone and olanzapine. Only $1 \%$ were treated with haloperidol, and $8 \%$ of patients did not follow the therapeutic instructions. The cardiac and metabolic side-effects of atypical antipsychotics must be taken into account when deciding the best therapy for patients with other important cardiovascular risk factors. It is necessary to take into consideration that some frequent side effects associated with olanzapine-based treatment include hyperglycemia, hypercholesterolemia and hypertriglyceridemia. Other less frequently reported side effects include the onset/exacerbation of diabetes.(12)

Atypical antipsychotics (risperidone, paliperidone the primary active metabolite of risperidone, olanzapine, clozapine and quetiapine) show preferential affinity for serotonin 5HT2A receptors versus dopamine D2 receptors, but the affinity differs between compounds. Neurons located in medial prefrontal cortex that express $5 \mathrm{HT} 2 \mathrm{~A}$ receptors project to the ventral tegmental area (VTA). The stimulation of these receptors promotes the release of dopamine in VTA and the appearance of specific symptoms of schizophrenia. 5HT2A antagonists, such as atypical antipsychotics, decrease dopamine release and improve symptoms.(13)

The low affinity for D2 receptors explains the different pharmaco-toxicological profile of these agents compared to classic antipsychotics (e.g. haloperidol), being free of extrapyramidal and endocrine side effects (except risperidone which induces hyperprolactinemia). The increased affinity of these compounds for serotonergic receptors contributes to the orexigenic effect, increased food intake, and body weight gain. Obesity predisposes to cardiovascular disease and diabetes. Adipose tissue secretes adipokines (leptin and adiponectin), cytokines that regulate the atherogenic process. In obese patients, a change in leptin/adiponectin ratio was observed, with an increased leptin level and atherosclerosis risk. For a review see.(14) Moreover, this class of antipsychotics has been reported to prolong the QT interval due to the high potential for blocking hERG potassium channels, increasing the risk of torsade de pointes or sudden death. Cases of myocarditis and cardiomyopathy have also been reported after quetiapine and risperidone treatment.(15)

Aripiprazole is another modern antipsychotic, but its pharmacological profile is different. This compound acts as a partial D2 receptor agonist and 5HT2A receptor antagonist. It acts as a D2 receptors antagonist only in areas where dopamine is in excess (in the mesolimbic pathway) without influencing dopaminergic neurotransmission in nigrostriatal and tuberoinfundibular pathways. This explains why the extrapyramidal and endocrine side effects are lower than in the case of classic antipsychotics.(16) In addition, aripiprazole has a weak/moderate torsadogenic risk and does not cause cardiomyopathy and myocarditis.(17)

Amisulpride is a less commonly used antipsychotic. It has an increased affinity for D2 and D3 dopaminergic receptors and is effective in reducing both positive and negative symptoms of schizophrenia. The action of the compound is dual: at low doses promotes dopaminergic neurotransmission due to the blockade of presynaptic dopaminergic receptors and at high doses it decreases dopaminergic neurotransmission by blocking postsynaptic dopaminergic receptors. Although it acts as a dopaminergic antagonist, the extrapyramidal side effects are lower compared to conventional antipsychotics.(18)

Recently, respiratory side effects of antipsychotics such as acute respiratory failure have also been described in literature, side effects reported as well by our patients.(19)

We noticed in our study the frequent use of benzodiazepines as an adjuvant treatment, especially alprazolam and clonazepam. Although both compounds are benzodiazepines derivatives, their indications are different. Alprazolam is used as an anxiolytic while clonazepam is used, usually, as an anticonvulsant. The effectiveness of benzodiazepines as adjunctive treatment in schizophrenia has been speculated for more than 70 years but it is not in accordance with their classic indication. Stimulation of $\mathrm{GABA}_{\mathrm{A}}$ receptors by benzodiazepines appears to prevent the presynaptic release of dopamine in the mesolimbic region and delays the adaptation of dopaminergic neurons to neuroleptics.(20)

In some patients, in case of treatment cessation (voluntarily or recommended) dopamine supersensitivity psychosis (DSP) and dyskinesia occur, possibly due to upregulation of D2 receptor density, as a consequence of longterm blockade of D2 receptors. Studies have shown that the development of DSP is correlated with altered GABA-ergic neurotransmission. Clonazepam, as a potent agonist of $\mathrm{GABA}_{\mathrm{A}}$ receptors, can effectively prevent the onset of extrapyramidal disorders.(21)

\section{CONCLUSIONS}

Schizophrenic patients with co-occurring somatic conditions have major impairment in functioning in life roles (education, social, family life).

Personality disorders can worsen the evolution of schizophrenic patients and furthermore the outcome of those patients that present co-occurring somatic disorders.

Excess mortality of patients with schizophrenia can be associated with the high prevalence of cardiovascular diseases encountered in this group of patients. Healthcare providers should take into consideration the fact that psychiatric patients sum up multiple modifiable risk factors and the onset of somatic illnesses may occur earlier in their life.

Antipsychotic treatment should be individualised so that the overall risk for somatic conditions can be minimised. Periodic laboratory examinations should be performed in order to detect and treat any metabolic abnormality.

Further research is required in order to investigate the onset of somatic comorbidities and to establish an advanced management for this particular group of schizophrenic patients.

\section{REFERENCES}

1. Šimunović Filipčić I, Filipčić I. Schizophrenia and Physical Comorbidity. Psychiatr Danub. 2018 Jun;30(Suppl 4):152157

2. Laursen TM, Munk-Olsen T, Gasse C. Chronic somatic comorbidity and excess mortality due to natural causes in persons with schizophrenia or bipolar affective disorder. PLoS One. 2011;6(9):e24597

3. Salcudean A. The autolitic risk in patients with schizophrenia. Acta Medica Transilvanica. 2011;16(3):481483

4. Salcudean A, Sirbu E, Muresan D, Bodo C. Particular aspects of social integration among patients with schizophrenia. Acta Marisiensis - Seria Medica. 2021;67(1): 34-38

5. Salcudean A, Ferencz M, Sabau D, Gherman M. Clinical evolution of patients with schizophrenia associated with 
addictive disorders. Acta Marisiensis - Seria Medica. 2020;66(4):152-154.

6. Siafis S, Tzachanis D, Samara M, Papazisis G. Antipsychotic Drugs: From Receptor-binding Profiles to Metabolic Side Effects. Curr Neuropharmacol. 2018;16(8):1210-1223.

7. Dieset I, Andreassen OA, Haukvik UK. Somatic Comorbidity in Schizophrenia: Some Possible Biological Mechanisms Across the Life Span. Schizophr Bull. 2016 Nov;42(6):1316-1319.

8. Ochoa S, Usall J, Cobo J, Labad X, Kulkarni J. Gender differences in schizophrenia and first-episode psychosis: a comprehensive literature review. Schizophr Res Treatment. 2012;2012:916198

9. Lehrer DS, Lorenz J. Anosognosia in schizophrenia: hidden in plain sight. Innov Clin Neurosci. 2014 May;11(5-6):107.

10. Simonsen E, Newton-Howes G. Personality Pathology and Schizophrenia. Schizophr Bull. 2018 Oct 17;44(6):11801184

11. Howell S, Yarovova E, Khwanda A, Rosen SD Cardiovascular effects of psychotic illnesses and antipsychotic therapy. Heart (British Cardiac Society). 2019 Dec;105(24):1852-1859.

12. Farwell WR, Stump TE, Wang J, Tafesse E, L'Italien G, Tierney WM. Weight gain and new onset diabetes associated with olanzapine and risperidone. J Gen Intern Med. 2004 Dec;19(12):1200-5.

13. Bortolozzi A, Díaz-Mataix L, Scorza MC, Celada P, Artigas F. The activation of 5-HT receptors in prefrontal cortex enhances dopaminergic activity. J Neurochem. 2005 Dec;95(6):1597-607.

14. Reponen EJ, Tesli M, Dieset I, Steen NE, Vedal TSJ, Szabo A, et al. Adiponectin Is Related to Cardiovascular Risk in Severe Mental Illness Independent of Antipsychotic Treatment. Front Psychiatry. 2021 May 28;12:623192.

15. Stoner SC. Management of serious cardiac adverse effects of antipsychotic medications. Ment Health Clin. 2018 Mar 23;7(6):246-254.

16. Tuplin EW, Holahan MR. Aripiprazole, A Drug that Displays Partial Agonism and Functional Selectivity. Curr Neuropharmacol. 2017 Nov 14;15(8):1192-1207.

17. Polcwiartek C, Sneider B, Graff C, Taylor D, Meyer J, Kanters JK, et al. The cardiac safety of aripiprazole treatment in patients at high risk for torsade: a systematic review with a meta-analytic approach. Psychopharmacology (Berl). 2015 Sep;232(18):3297-308

18. Mota NE, Lima MS, Soares BG. Amisulpride for schizophrenia. Cochrane Database Syst Rev. 2002;2002(2):CD001357.

19. Wang MT, Lin CW, Tsai CL, Wang YH, Lai JH, Yeh CB, et al. Use of antipsychotics and the risk of acute respiratory failure among adults: A disease risk score-matched nested case-control study. $\mathrm{Br} \quad \mathrm{J}$ Clin Pharmacol. 2020 Nov;86(11):2204-2216.

20. Gaillard R, Ouanas A, Spadone C, Llorca PM, Lôo H, Baylé FJ. Benzodiazépines et schizophrénie, revue de la littérature [Benzodiazepines and schizophrenia, a review of the literature]. Encephale. 2006 Nov-Dec;32(6 Pt 1):100310. French.

21. Fukai M, Hirosawa T, Takahashi T, Kaneda R, Kikuchi M, Minabe Y. Clonazepam improves dopamine supersensitivity in a schizophrenia patient: a case report. Ther Adv Psychopharmacol. 2017 Mar;7(3):113-117. 